\title{
Dynamics simulation studies of solvation effect on the trans-xylomollin conformation
}

\author{
Radia Mahboub \\ Department of Chemistry, Faculty of Sciences, University of Tlemcen, B.P. 119, \\ Tlemcen 13 000, Algeria. \\ E-mail address: radiamahboub@yahoo.com
}

\begin{abstract}
The present work describes the solvation effect on the trans-xylomollin conformation. We have studied the trans-xylomollin conformations with the distance restraints using simulation calculations. Distance Restraint Molecular Dynamic (DR-MD) and Distance Restraint Langevin Dynamic (DR-LD) simulations of the trans-xylomollin were performed with an efficient program. The geometries, interaction energies, bonds, angles, and the Van der Waals (VDW) interactions were carried out in solution and gas phases. This comparative study shows that the trans-xylomollin acquires low total energy in solution using DR-MD method and stable conformation under the AMBER field. This molecule reaches its high stable conformation state in solution environment. The solvation effect is more important with DR-MD simulations. Our results are goods and in agreement with the used force field.
\end{abstract}

Keywords: Conformation; Solvation; Distance Restraints; Dynamics Simulations

\section{INTRODUCTION}

The movements of solute in solution are essentially depending on the stability between solute-solvent and solvent-solvent interactions. The chosen solvent determines the solvation of molecules, the protonation condition, and the conformation for large flexible molecules. The real structure of biological macromolecules such as proteins is governed by interactions with water [1,2]. Biological macromolecules and bioactive molecules are influenced by water [3-5]. The condition to accomplish their function correctly, these molecules have needed to be under intrinsic conditions to adopt their real conformational structure [6-10]. In addition, the conformations of solute are influenced by the surrounding water molecules. Because the restricted conformational of water, the dynamics of solute is diminished. Thus, modeling and dynamics of solute in solution generally requires the consideration of interactions with solvent molecules. In usual Molecular dynamics (MD) simulations of solute-water systems, water molecules are described explicitly in atomic detail employing an atom-based molecular force field. Such MD simulations involve frequently a large number of atoms, where most of them belong to solvent molecules. The computational costs of such molecular systems increase faster, making MD simulations with explicit solvent models somewhat expensive [11].

Diverse methods have been applied to reduce the time required for MD simulations. Most important one is the use of implicit solvent models for water which diminish the number 
of atoms [12-20]. Another efficiency advantage of implicit solvent models is the absence of solvent viscosity. As a consequence, the used time of MD simulations with an implicit solvent model becomes fast. This allows exploring the conformational space of a solute molecule in little simulation time. Water interacts with solute molecules in three ways. The two direct type of interaction are electrostatic [21] and Van der Waals (VDW) interactions [22] which include the hydrogen bonds. The one indirect type is due to the hydrophobic effect [2, 23-27]. Langevin Dynamics happen in the study of solvent effect and require stochastic solvent environment with atomic temperature control. The implicit solvent LD simulation can use small number of particles and gives large scale conformation. Because it is difficult to follow the motion of real systems to reach the global minimum, the energy surface is less effective to motion.

In the present work, we describe and characterize the molecular structure of transxylomollin by Distance Restraints Molecular and Langevin dynamics (DR-MD and DR-LD) simulations using AMBER as force field. We discuss the computational chemistry results of the trans-xylomollin compound then we compare the two calculation DR-MD and DR-LD methods. In section 2, we describe the materials and methods used in this study. In the next section, we have detailed the molecular calculations: i) dynamic simulations, ii) geometry optimization and iii) conformation representation. First, we describe the evolution of:

i) energies, and ii) temperatures in both vacuum and water environments by DR-MD and DRLD calculations, followed by comparison with MD and LD calculations. Then, we discuss the calculation results obtained with distance restraints using geometry optimization, and then we compare all these results with those obtained without restraints. Finally, we estimate the torsion angle in terms of distance after each dynamics simulation. In the last section, we give the conclusion.

\section{MATERIALS AND METHODS}

Molecular dynamics (MD) is a useful technique to study the kinetics and thermodynamics properties of macroscopic systems involving a few molecules such as biomolecules, heterocyclic compounds, proteins and other macromolecules in order to provide diffusion [28, 29], thermodynamic and structural [29, 30], conformational properties $[32,33]$ and structure-activity relationship [34]. The success of MD is the study process energy of organic compounds as protein, heterocyclic, and important conformational changes in a small time step size.

Solvent effects can be modeled by Langevin dynamics then a molecular system in a liquid environment can be studied. These simulations can be much faster than molecular dynamics. Langevin dynamics simulates the effect of molecular collisions and the resulting dissipation of energy that occurs in real solvents, without explicitly including solvent molecules. This is accomplished by adding a random force and a frictional force to each atom at each time step $[35,36]$. Because of the implicit treatment of the solvent, this method is particularly well-suited for studying large molecules in solution [37].

In gas, the system was simulated using Molecular and Langevin dynamics (MD and LD) with $0.0001 \mathrm{ps}$ step. For the LD simulation, the frictional coefficient was chosen to 0.05 $\mathrm{ps}^{-1}$. Temperature was kept constant at $300 \mathrm{~K}$. The starting temperature was taken at $100 \mathrm{~K}$ and the step at $20 \mathrm{~K}$. In water simulations, the system was placed in a box $(20 \times 20 \times 20 \AA)$ containing one molecule of trans-xylomollin and 246 water molecules and cut-off $4 \AA$. 
Optimization of the molecule was realized in periodic boundary conditions (PBC). The compound was solvated by added water molecules. The systems were first energy minimized steps with the conjugate gradient algorithm. Then, the position-restrained MD simulation was run 0.5ps. Distance Restraint MD and LD simulations (DR-MD; DR-LD) were run with the same parameters as the MD and LD simulations. The $\mathrm{d}_{\mathrm{H} 29-\mathrm{H} 37}(\mathrm{D})$ distance was varying from 3 to $6 \AA$ with $1 \AA$ as increment.

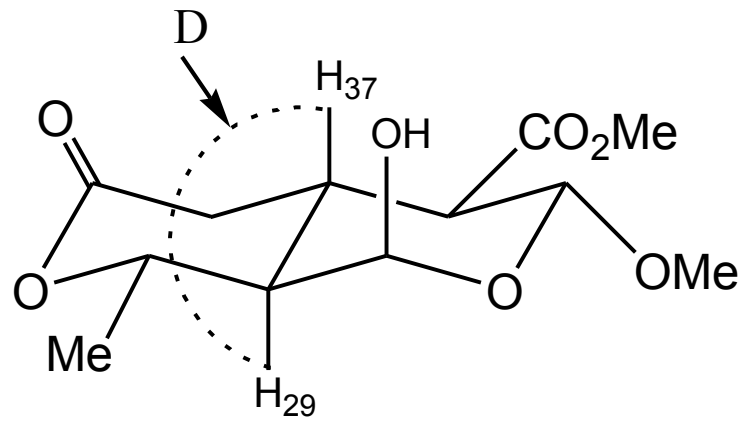

Scheme 1. Distance Restraint $\left(\mathrm{d}_{\mathrm{H} 29-\mathrm{H} 37}\right)$ representation in trans-xylomollin.

\section{RESULTS AND DISCUSSIONS}

Primary analysis of dynamics simulations (MD and LD) presented here showed that for all experiments temperature was constant, which showed that the experiments were carried out correctly. The next stage involved the development of comparative studies on the realized DR-MD and DR-LD simulations with MD and LD calculations. The energy properties obtained by MM are analyzed for each dynamics method. Finally to check the solvent effect, the conformational structure was studied by the evolution of torsion angle in terms of distant restraint for the trans-xylomollin.

\section{1. Dynamics simulations with distance restraints}

Dynamic simulations (MD and LD) were accomplished at constant temperature, using AMBER force field. The conjugate gradient algorithm was preferred because for the constant dielectric. We have calculated the trans-xylomollin dynamic properties in both gas and water. We have employed the TIP3P water molecules model, and chosen the bath relaxation time equals to $0.1 \mathrm{ps}$. The simulation temperature was fixed to $300 \mathrm{~K}$ and the step at $20 \mathrm{~K}$. The run time was $0.5 \mathrm{ps}$, the step size was $0.0001 \mathrm{ps}$ and the heat time was $0.1 \mathrm{ps}$. In LD simulations, the coefficient of friction was to $0.05 \mathrm{ps}^{-1}$. Distance Restraint MD and LD simulations were run with the same parameters as the dynamics simulations. We have varying the distance restraint D from 3 to $6 \AA$ with an internal force constant of $5 \mathrm{Kcal} / \AA^{2}$ in both gas and water.

The distance $\mathrm{D}\left(\mathrm{d}_{\mathrm{H} 29-\mathrm{H} 37}\right)$ was taken as the distance between 1, 4 non-bonded atoms: $\mathrm{H}_{29}$, and $\mathrm{H}_{37}$. Here, we study the evolution of: i) energies, ii) energy deviations and iii) temperatures in terms of distance in both vacuum and water environments for the transxylomollin.

At first, we have calculated average energies for the studied system then represented them in Figures 1. The average energies are in good agreement with the simulation accuracy. The sampling results of step-size of DR-MD and DR-LD methods in vacuum and water are 
presented in Figures (A) and (B), respectively. For the trans-xylomollin, the evolutions of average energies: total and potential (respectively ETOT and EPOT) are different in gas and water environments with DR-MD and DR-LD. In gas, ETOT and EPOT increase gradually with the distance.
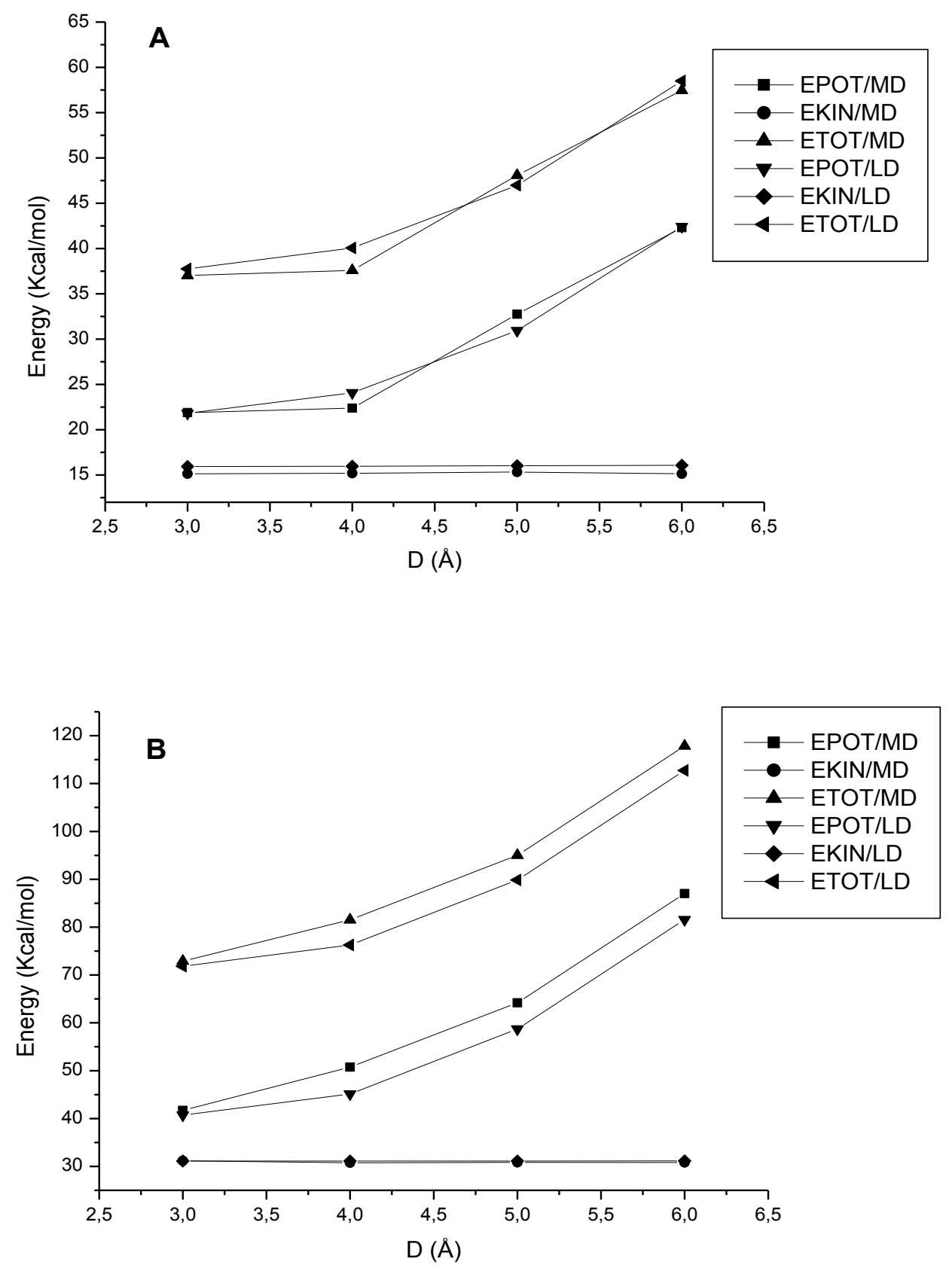

Fig. 1. Evolution of energies for trans-xylomollin with distance restraints using MD and LD simulations: (A) in gas and (B) in water.

The kinetic energies (EKIN) stay constants around $16 \mathrm{Kcal} / \mathrm{mol}$ (Figure 1A). In solution, we observe that all energies are multiplied by two if compared with those obtained in gas environment. So, we remark that the ETOT and EPOT values in DR-MD simulation 
are higher than in DR-LD one, and progress rapidly. In the same time, the kinetic energies provide an opposite situation i.e. they keep on stable during all simulations $(30 \mathrm{Kcal} / \mathrm{mol})$ (Figure 1B). So, kinetic energy is not affected by the distance restraints which have an effect mainly on the total and potential energies. The solvation energy in DR-MD simulation is greater than in DR-LD one.

Comparing the present results with those obtained after using Molecular and Langevin dynamics simulations without distance restraints, we note that the average energies calculated under DR-MD and DR-LD simulations are higher than these obtained by MD and LD techniques for both environments (Table 1). EPOT, EKIN and ETOT are comparable in both simulations. When using the LD simulation in water, the situation changes as of the use of implicit solvent; so energies increase largely. Here, we have illustrated the energy of the solvation $\mathrm{E}_{\mathrm{sol}}$ as the variation between $\mathrm{ETOT}_{\mathrm{xylo} \text {,solv }}$ and $\mathrm{ETOT}_{\mathrm{xylo} \text {,isol. }}$ So, the solvation effect is apparent in LD simulation and the energy of solvation is evaluated to $34.262 \mathrm{Kcal} / \mathrm{mol}$ (MD: $0.966 \mathrm{Kcal} / \mathrm{mol}$ ).

The situation is changed when using restraints. At the position of $3 \AA$, the energy of solvation is important with distance restraint for MD simulation (without DR: 0.966, with DR: $35.624 \mathrm{Kcal} / \mathrm{mol}$ ) but keeps constant for LD one (without DR: 34.262 , with DR: 34.076 $\mathrm{Kcal} / \mathrm{mol}$ ). As a result, the solvation phenomenon rises with the distance restraint under MD simulations, while it unchanged under LD ones. From 3 to $6 \AA$, the plotted curves show that the solvation energy obtained under DR-MD simulations grow up rapidly than the DR-LD ones (Figure 2).

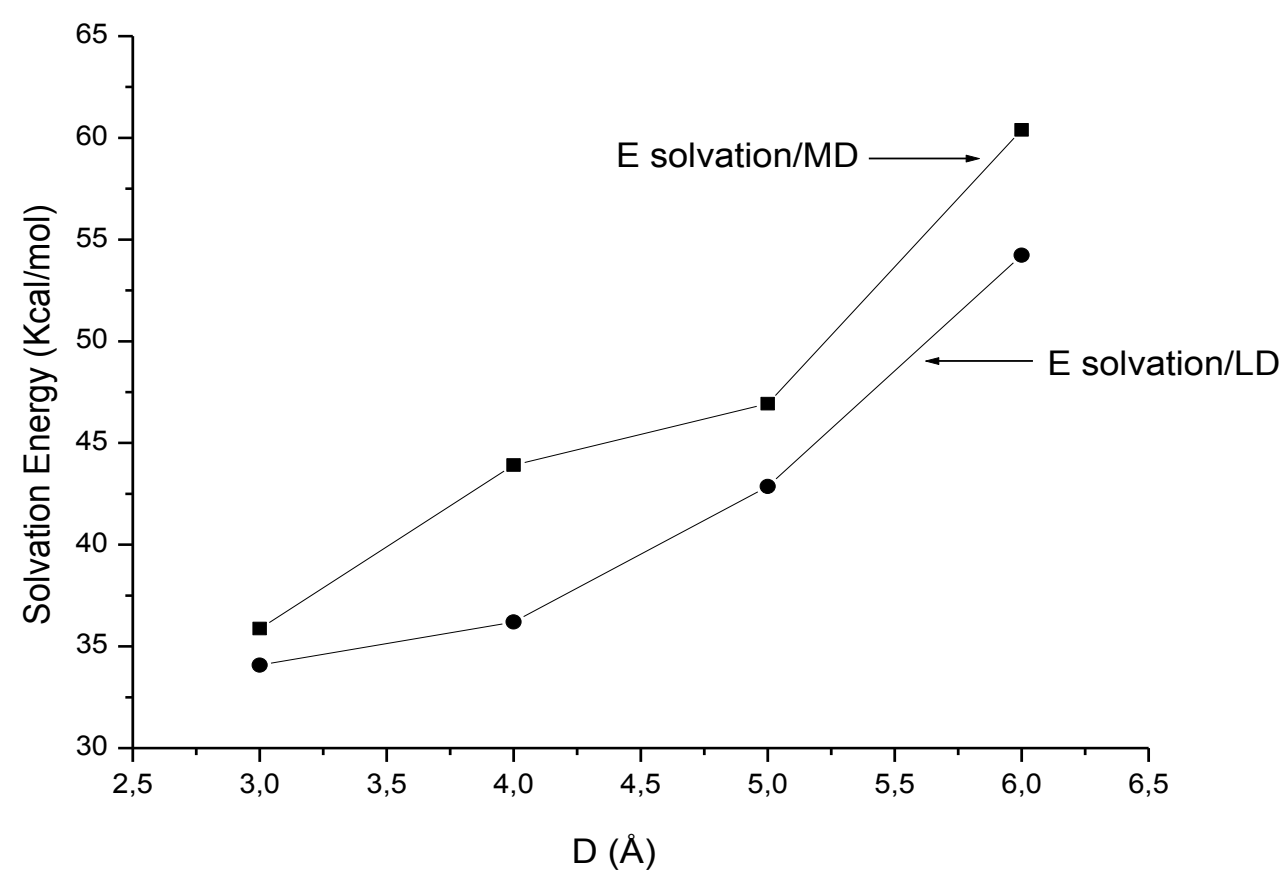

Fig. 2. Solvation energies of trans-xylomollin with distance restraints using MD and LD simulations. 
Table 1. Calculated properties of trans-xylomollin at $300 \mathrm{~K}$ using MD and LD simulations without distance restraints.

\begin{tabular}{|c|c|c|c|c|}
\hline Method & State & $\begin{array}{c}\text { EPOT } \\
(\text { Kcal/mol })\end{array}$ & $\begin{array}{c}\text { EKIN } \\
(\text { Kcal/mol })\end{array}$ & $\begin{array}{c}\text { ETOT } \\
(\mathbf{K c a l} / \mathbf{m o l})\end{array}$ \\
\hline \multirow{2}{*}{ MD } & Isolated & 19.5005 & 15.4537 & 34.9578 \\
\cline { 2 - 5 } & Solvated & 20.7647 & 15.1594 & 35.9242 \\
\hline \multirow{2}{*}{ LD } & Isolated & 21.7817 & 15.9100 & 37.6918 \\
\cline { 2 - 5 } & Solvated & 40.7749 & 31.1793 & 71.9542 \\
\hline
\end{tabular}

In the present simulation experiments, the temperature was changed. In vacuum, the fluctuations are important and the temperature increases until reaches the maxima at $139 \mathrm{~K}$ then decreases to $137 \mathrm{~K}$ for DR-MD simulation. At the same time and during DR-LD simulation, the temperature has a tendency to be constant $(144 \mathrm{Kcal} / \mathrm{mol})$ (Figure 3A). In water, the situation is much better.

We observe that the resulting trajectories are different: stable and attain the value of 282 $\mathrm{K}$ for DR-LD method but fluctuate then tend to stabilized at $279 \mathrm{~K}$ (Figure 3B). As consequences, the DR-MD and DR-LD simulations are more stable and keep being constant near the $300 \mathrm{~K}$ in water due to the interactions of water molecules.

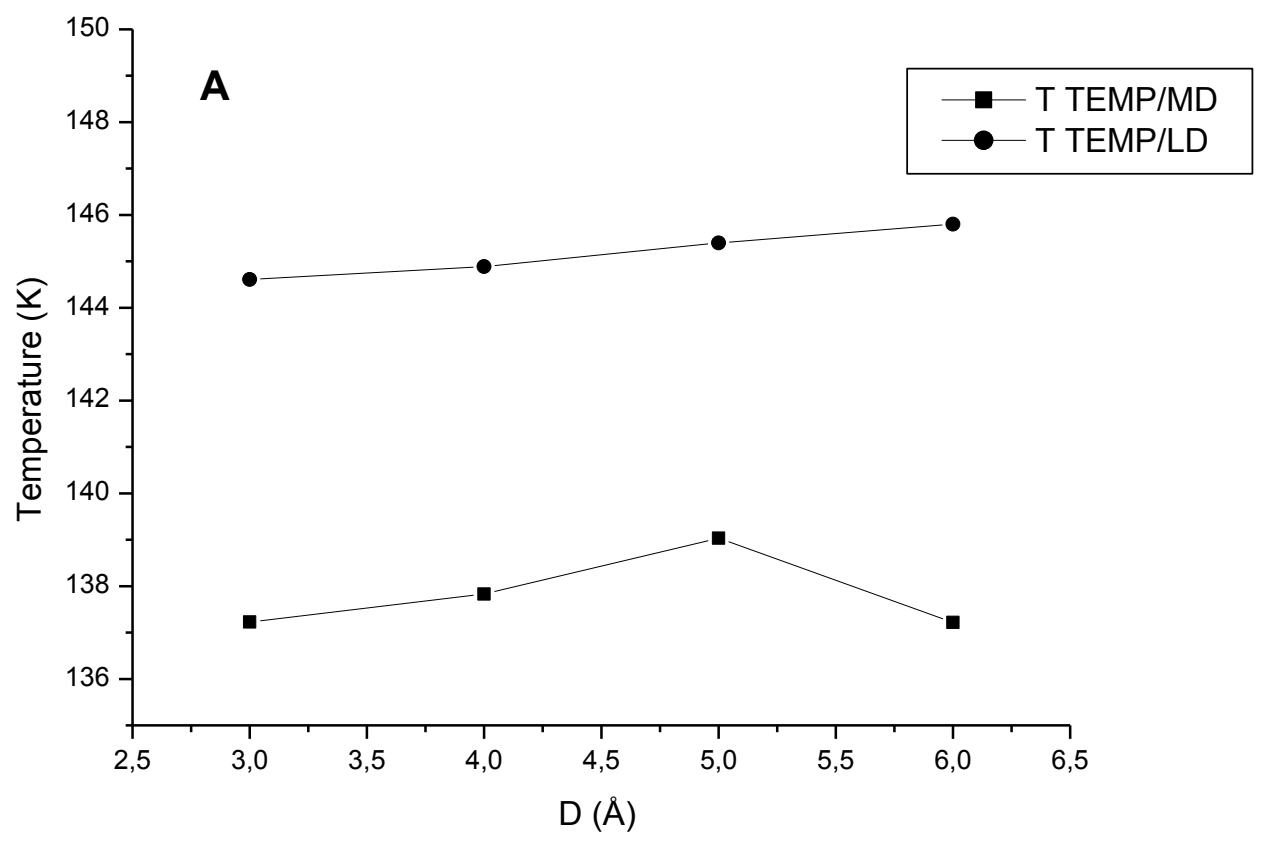

Fig. 3. Evolution of temperatures for trans-xylomollin with distance restraints using MD and LD simulations: (A) in gas and (B) in water. 


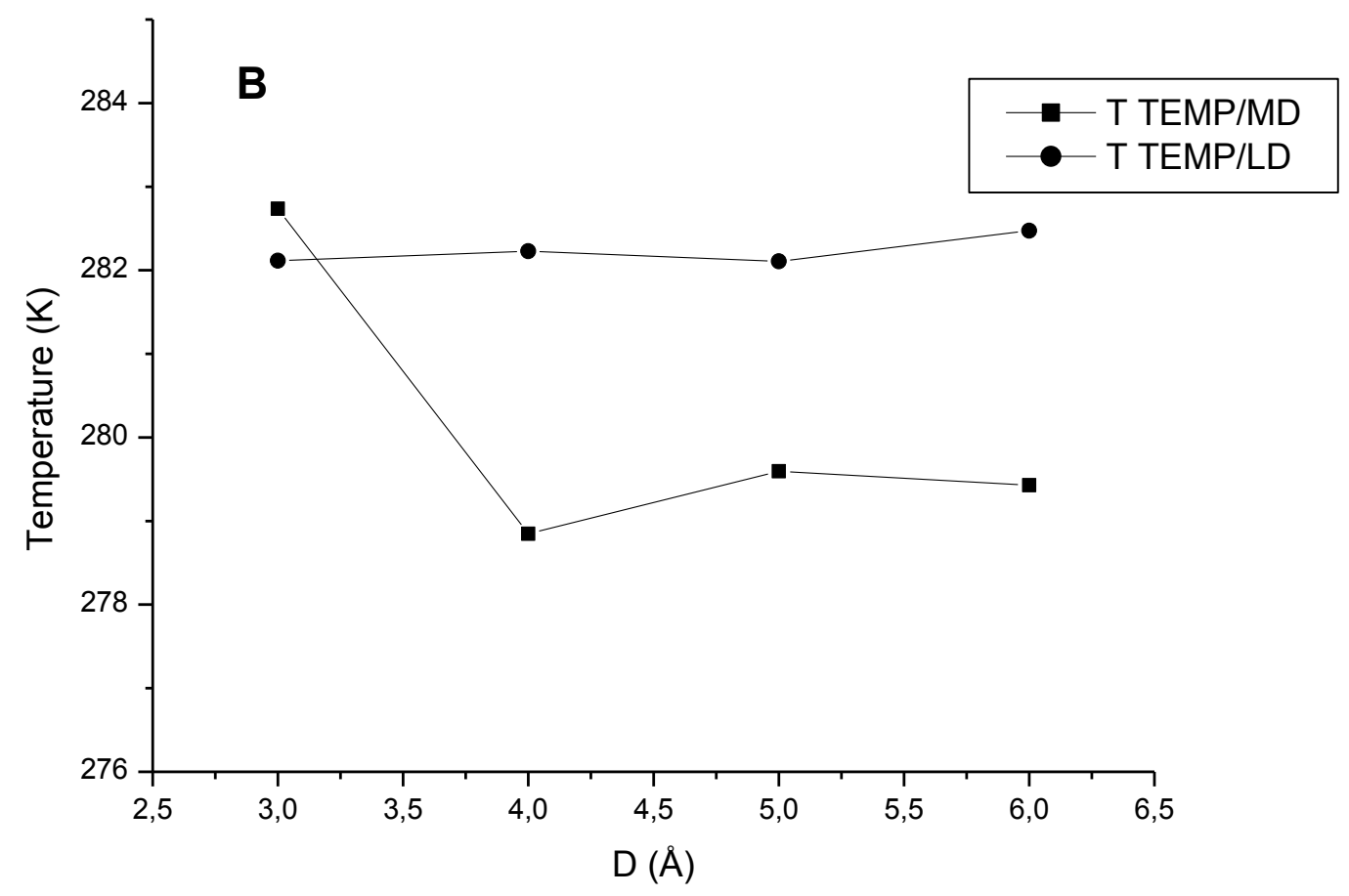

Fig. 3 (continue). Evolution of temperatures for trans-xylomollin with distance restraints using MD and LD simulations: (A) in gas and (B) in water.

\section{2. Molecular mechanic simulations with distance restraints}

For all simulations (DR-MM, DR-MD and DR-LD), the minimum total energies ET min calculated for the geometry optimization using AMBER are increasing with the distance in both environments.

We note that the dihedral factor has the large effect on minimizing total energy and keeps constant (around $9 \mathrm{Kcal} / \mathrm{mol}$ ) except for DR-MD calculations. For the last situation, we consider that this factor affects efficiently the system: the torsion angle of the transxylomollin molecule tends to change the conformation. We have plotted these results and reported the trajectories in Figures 4 and 5.

We note that the curves of the bond and angle progress slowly. For Van der Waals interactions, we remark that the energies seem similar in MM and LD calculations (3 $\mathrm{Kcal} / \mathrm{mol})$ and change in MD simulation and become little in water $(2.5 \mathrm{Kcal} / \mathrm{mol})$. After comparing all the minimum total energies (ETmin) obtained under distance restraints conditions by different calculations methods (DR-MM, DR-MD and DR6LD), we have done the following observations.

In gas, the DR-MM and DR-LD curves seem to be identical and the minimum value of energy is obtained at $4 \AA$ when using DR-MD simulations (see respectively Figures 4A, 4C and 4B). 

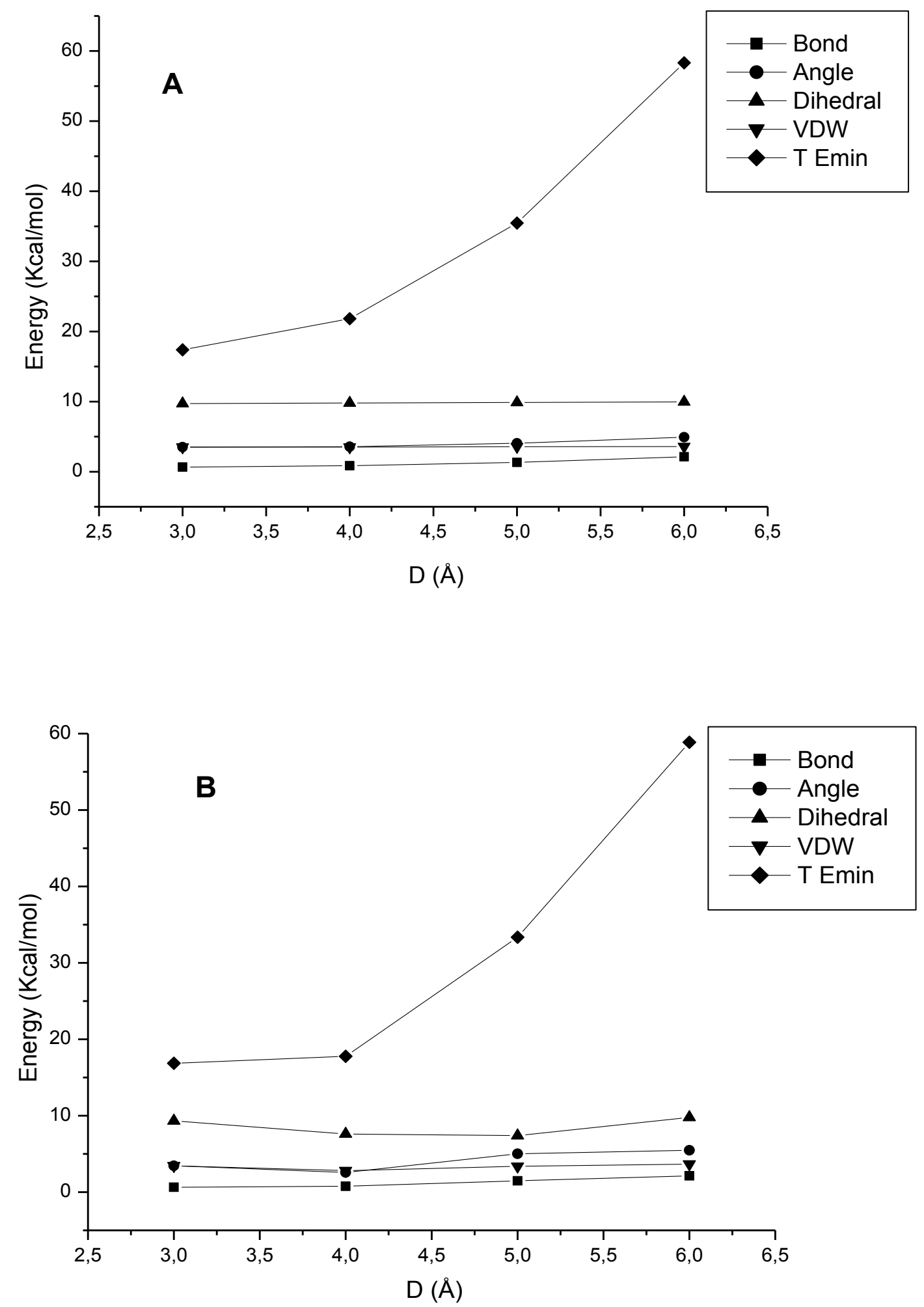

Fig. 4. Evolution of optimized geometry energies for trans-xylomollin in gas with distance restraints using: (A) MM, (B) MD and (C) LD. 


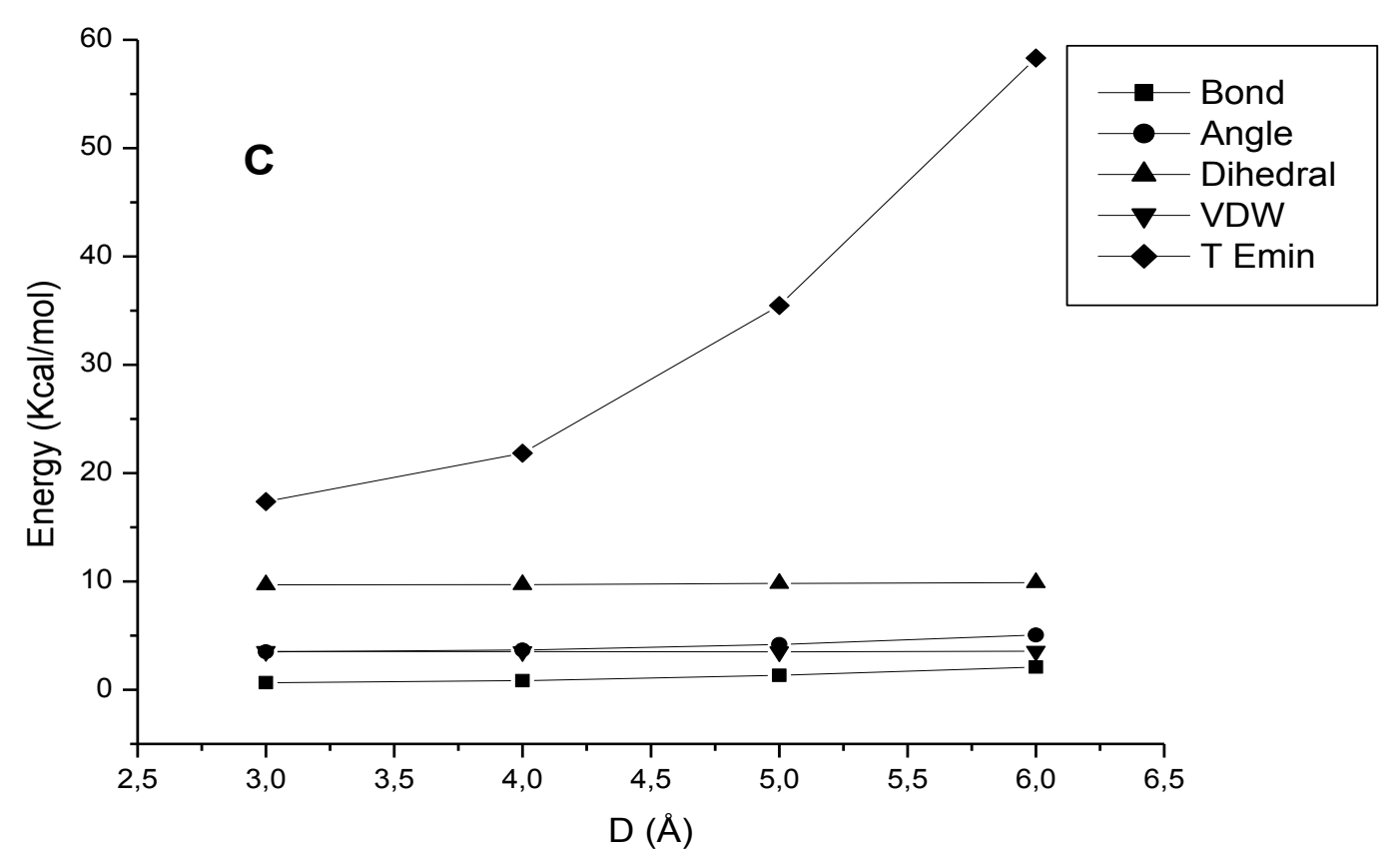

Fig. 4 (continue). Evolution of optimized geometry energies for trans-xylomollin in gas with distance restraints using: (A) MM, (B) MD and (C) LD.

The situation changes in solution. From $3 \AA$ until $5 \AA$, the small energies are obtained by DR-MD calculations; but at the high D value ( $6 \AA$ ), the high energy concerns the LD method. Consequently, our system is much stable in water with DR-MD simulation in the distance interval of $[3,5 \AA]$ (see respectively Figures $5 \mathrm{~B}$ and $5 \mathrm{C}$ ).

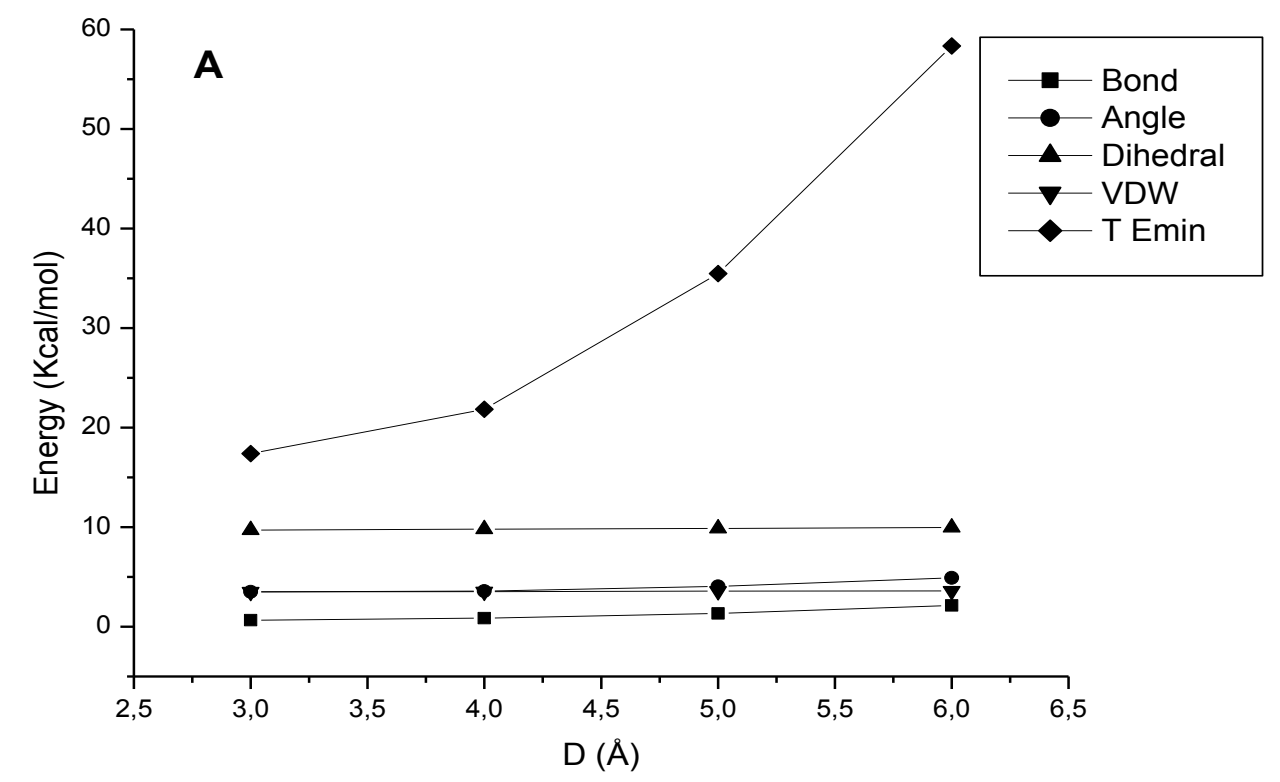

Fig. 5. Evolution of optimized geometry energies for trans-xylomollin in solution with distance restraints using: (A) MM, (B) MD and (C) LD. 

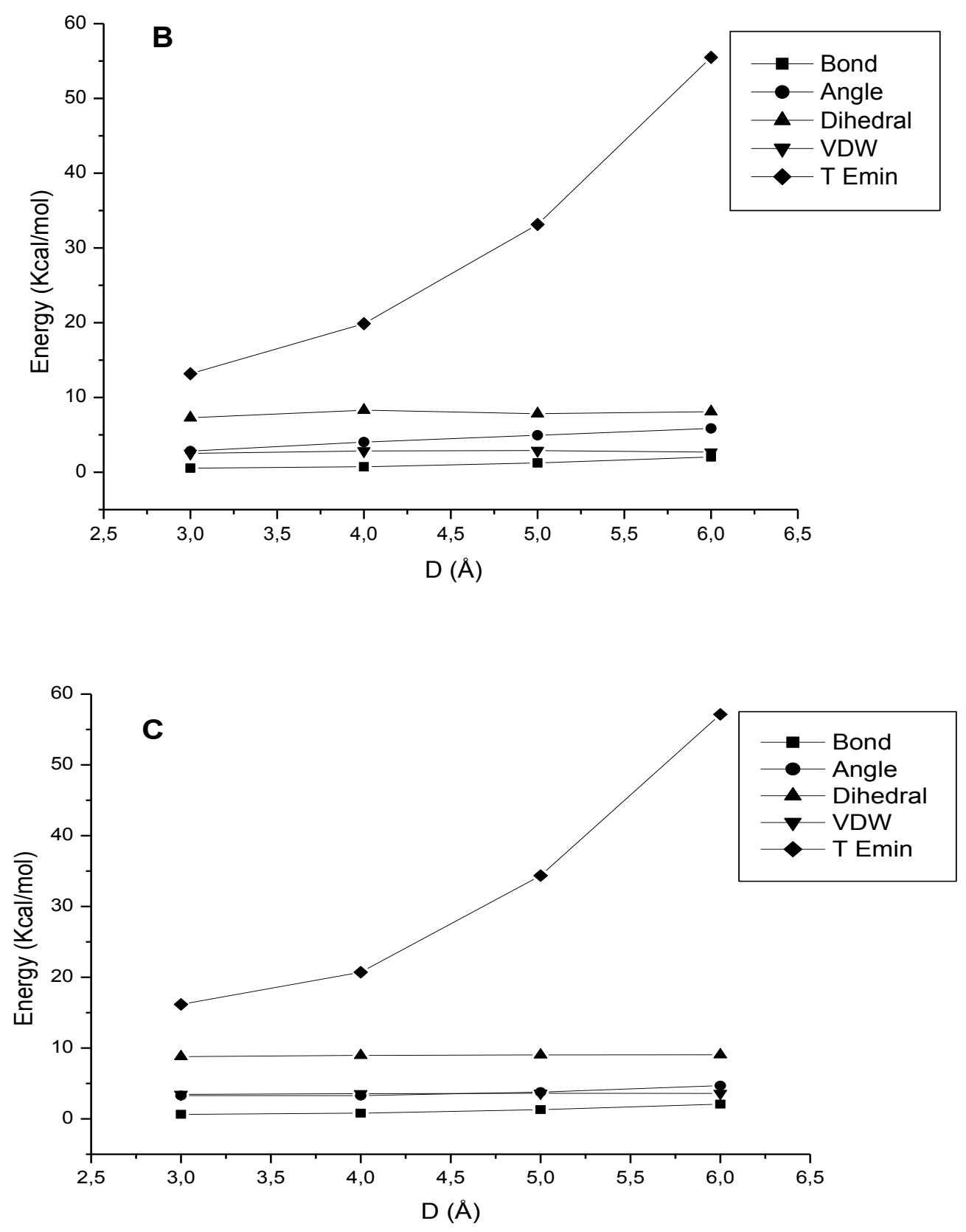

Fig. 5 (continue). Evolution of optimized geometry energies for trans-xylomollin in solution with distance restraints using: (A) MM, (B) MD and (C) LD.

In both environments and without restraints, the minimum total energies (ET min) calculated for the geometry optimization using MM method under AMBER are similar and are the much higher ones than MD and LD (respectively in gas: $17.375 \mathrm{Kcal} / \mathrm{mol}$ and in water: $17.382 \mathrm{Kcal} / \mathrm{mol}$ ). We note that the dihedral factor has the large effect on minimizing total energy (Table 2). For the last situation, we consider that this factor affects efficiently the system: the conformation of the trans-xylomollin molecule tends to change. Moreover, the angle (which represents the bend) and Van der Waals (as dipole-dipole interactions) energies are similar. 
In general, we observe that in MD simulations the evolution of energy properties stay unaffected for both environments by comparing them to LD one. At this point, the increased values are obtained in solution environment. The exception is the dihedral factor which is not affected by the nature of the surrounding. Finally, our system is more stable when working under AMBER (as force field) and employing the MD simulations.

Table 2. Geometry optimization properties of trans-xylomollin at $300 \mathrm{~K}$ using MM, MD and LD simulations.

\begin{tabular}{|c|c|c|c|c|c|c|}
\hline Method & State & $\begin{array}{c}\text { Bond } \\
\text { (Kcal/mol) }\end{array}$ & $\begin{array}{c}\text { Angle } \\
\text { (Kcal/mol) }\end{array}$ & $\begin{array}{c}\text { Dihedral } \\
\text { (Kcal/mol) }\end{array}$ & $\begin{array}{c}\text { VDW } \\
\text { (Kcal/mol) }\end{array}$ & $\begin{array}{c}\text { Energy } \\
\text { (Kcal/mol) }\end{array}$ \\
\hline \multirow{2}{*}{ MM } & Isolated & 0.6607 & 3.5038 & 9.7106 & 3.4996 & 17.3746 \\
\cline { 2 - 7 } & Solvated & 0.6605 & 3.5034 & 9.7104 & 3.5079 & 17.3820 \\
\hline \multirow{2}{*}{ MD } & Isolated & 0.5663 & 2.7598 & 8.5234 & 2.9413 & 14.7907 \\
\cline { 2 - 7 } & Solvated & 0.5899 & 2.8498 & 8.4689 & 2.9680 & 14.8764 \\
\hline \multirow{2}{*}{ LD } & Isolated & 0.5774 & 2.7936 & 8.5331 & 2.8902 & 14.7941 \\
\cline { 2 - 7 } & Solvated & 0.6598 & 3.4546 & 8.5237 & 3.8446 & 16.4827 \\
\hline
\end{tabular}

\section{3. Conformation studies}

To provide a better estimate of conformations, we should search the conformational space in reasonable computing time. So, we run the simulations at the constant temperature for the chosen distance restraints, and then we run a geometry optimization on each structure. In table 3, we have grouping the following characteristics which are determined after geometry optimization (and dynamic simulations with distance restraints): the distance restraints, the calculated torsion angles and the conformations obtained after DR-MD and DRLD simulations. So, we have plotted torsion angle in terms of distances. Finally, we have compared representation structures to determine the best stable conformation.

Table 3. Torsion angles of trans-xylomollin at $300 \mathrm{~K}$ with distance restraints after DR-MD and DRLD simulations.

\begin{tabular}{|c|c|c|c|c|}
\hline \multirow{2}{*}{$\mathbf{D}(\AA)$} & \multicolumn{4}{|c|}{$\Phi\left(^{\circ}\right)$} \\
\cline { 2 - 5 } & \multicolumn{2}{|c|}{ Isolated } & \multicolumn{2}{c|}{ Solvated } \\
\cline { 2 - 5 } & MD & LD & MD & LD \\
\hline 3 & -165.816 & -164.397 & -179.964 & -166.454 \\
\hline 4 & -179.825 & -164.384 & 170.401 & -167.921 \\
\hline 5 & -164.920 & -164.909 & 164.646 & -167.971 \\
\hline 6 & -163.814 & -165.190 & 165.696 & -167.039 \\
\hline
\end{tabular}


From the calculated torsion angles and the representations (Table 3), we note that with DR-MD simulation and under $4 \AA$ the trans-xylomollin acquires its maxima anhedral angle in gas $\left(-179.825^{\circ}\right)$. In water, the torsion angle changes $\left(170.40^{\circ}\right)$. In these situations, the molecule reaches its high stable conformation states. So, the torsion angle does not be affected by distance under DR-LD method (Figures 6).
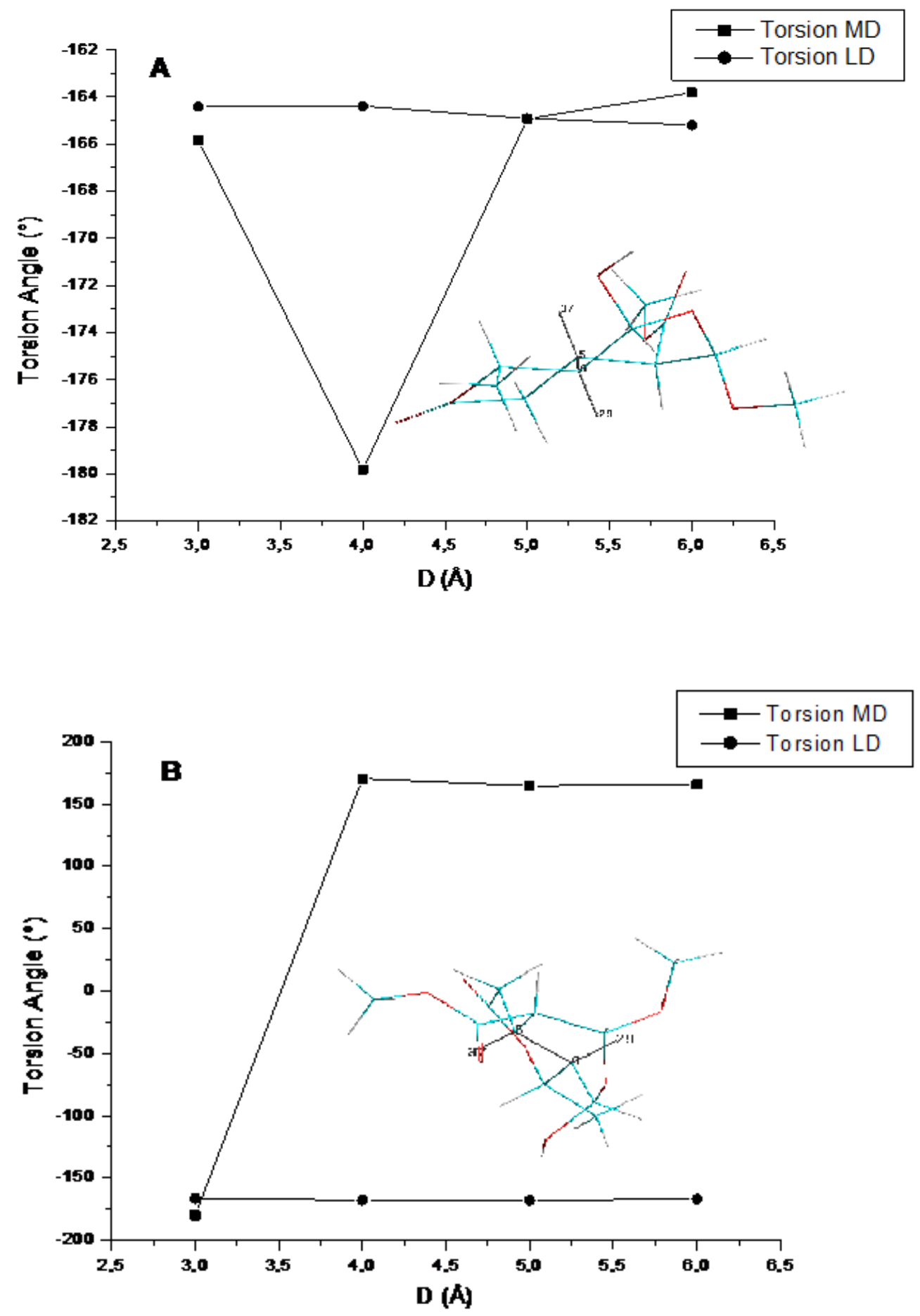

Fig. 6. Evolution of torsion angles for trans-xylomollin with distance using dynamics simulations: (A) in gas and (B) in water. 
In the two dynamics simulations, the molecule adopts anti position with low energies. With DR-MD simulation, the molecule has more stable conformations for $-179.825^{\circ}$ $\left(170.40^{\circ}\right)$ than DR-LD one. So, the distance restraints affect largely the torsion angles in solution when using MD calculation. In DR-MD simulation, significant value of the torsion ($179.825^{\circ}$ ) appears at $4 \AA$ in gas environment, referring to the most extended trans-xylomollin structure (i.e. anti conformer) (Figure 6A), while the high value change with larger distances (from 4 to $6 \AA$ ) (Figure $6 \mathrm{~B}$ ) in solution. At the distance of $4 \AA$, we note that the VDW energies are reduced in both environments under DR-MD method if compared to those in DRLD simulation. Consequently, the minimum total energies (ET min) are also decreasing.

The water molecules create a hydrophobic effect which affects largely the nonpolar hydrogen in the molecule and produce angle deformation $\left(170.40^{\circ}\right)$ mainly under DR-MD simulations. So, the 1-4 atom pair interactions between $\mathrm{H}_{29}$ and $\mathrm{H}_{37}$ reach it minima states which give favorable conformer structures. These ones are confirmed by the obtained torsion angles.

\section{CONCLUSIONS}

We have studied the distance restraint effects on the solvation properties for transxylomollin. We have chosen the DR-MD and DR-LD methods to predict much better these characteristics. The geometries, interaction energies, bonds, angles, and the VDW interactions were carried out in solution and in gas phase. We have calculated the thermodynamic and structural properties using AMBER as the force field.

In water, we note that the total energies obtained by DR-MD simulations are higher than those in gas. We conclude that the restraint effects are more efficient in water and suitable for the system, and give good results for force field. In the same environment, we remark that the temperature moves toward $300 \mathrm{~K}$. As consequence, the conformation is more stable in solution. We note that the geometry optimizations are affected by restraints in presence of water molecules. So, the solvation phenomenon is much important with the DR-MD simulations than the DR-LD simulations.

We conclude that our DR-MD simulations under AMBER field gives best results; the xylomollin acquires the low-energy at $4 \AA$ and more stable conformation mainly in water with an anti position $\left(170.4^{\circ}\right)$. So, the molecule reaches its high stable conformation state in solution environment.

\section{References}

[1] Ben-Naim A., Solvent Effects on Protein Stability and Protein Association, In ProteinSolvent Interactions, 1st ed.; Gregory, R. B., Ed.; Marcel Dekker, Inc.: New York, 1995; Vol. 592, pp 387-420.

[2] Water: A Comprehensive Treatise; Franks F., Ed.; Plenum Pub Corp: New York, 1972-1982; Vol. 1-7.

[3] Klebe G., Drug Discovery Today 11 (2006) 580-594.

[4] Corbeil C. R., Moitessier N., J. Chem. Inf. Model. 49 (2009) 997-1009.

[5] Froloff N., Windemuth A., Honig B., Protein Sci. 6 (1997) 1293-1301. 
[6] Eisenberg D., McLachlan A. D., Nature. 319 (1986) 199-203.

[7] Teeter M. M., Annu. Rev. Biophys. Biophys. Chem. 20 (1991) 577-600.

[8] Soares C. M., Teixeira V. H., Baptista A. M., Biophys. J. 84 (2003) 1628-1641.

[9] Privalov P. L., Makhatadze G. I., J. Mol. Biol. 232 (1993) 660-679.

[10] Honig B., Yang A. S., Free Energy Balance in Protein Folding. In Adv. Protein Chem.; Anfinsen C. B., Richards F. M., Edsall J. T., Eisenberg D. S., Eds.; Academic Press: San Diego, 1995; Vol. 46, pp 27-58.

[11] Juneja A., Numata J., Nilsson L., Knapp E. W., J. Chem. Theory Comput. 6 (2010) 1871-1883.

[12] Warwicker J., Watson H. C., J. Mol. Biol. 157 (1982) 671-679.

[13] Cramer C. J., Truhlar D. G., Chem. Rev. 99 (1999) 2161-2200.

[14] Roux B., Simonson T., Biophys. Chem. 78 (1999) 1-20.

[15] Gilson M. K., Davis M. E., Luty B. A., McCammon J. A., J. Phys. Chem. 97 (1993) 3591-3600.

[16] Baker N. A., Curr. Opin. Struct. Biol. 15 (2005) 137-143.

[17] Lopes A., Alexandrov A., Bathelt C., Archontis G., Simonson T., Proteins: Struct. Funct. Bioinf. 67 (2007) 853-867.

[18] Ferrara P., Apostolakis J., Caflisch A., Proteins: Struct. Funct. Bioinf. 46 (2002) 24-33.

[19] Chen J., Brooks C. L., Phys. Chem. Chem. Phys. 10 (2008) 471-481.

[20] Vorobjev Y. N., Almagro J. C., Hermans J., Proteins: Struct. Funct. Bioinf. 32 (1998) 399-413.

[21] Warshel A., Aqvist J., Annu. Rev. Biophys. Biophys. Chem. 20 (1991) 267-298.

[22] Dill K. A., Biochemistry. 29 (2002) 7133-7155.

[23] Ben-Naim A., Hydrophobic Interactions; Plenum Press: New York: 1980.

[24] Southall N. T., Dill K. A., Haymet A. D. J., J. Phys. Chem. B. 106 (2001) 521-533.

[25] Kauzmann W., Some Factors in the Interpretation of Protein Denaturation. In Adv. Protein Chem.; Anfinsen C. B., Anson M. L., Bailey K., Edsall J. T., Eds.; Academic Press: New York, 1959; Vol. 14, pp 1-63.

[26] Tanford C., Protein Denaturation. In Adv. Protein Chem., Anfinsen C. B., Anson M. L., Edsall J. T., Frederic M. R., Eds.; Academic Press: New York, 1968; Vol. 23, pp. 121-282.

[27] Hummer G., Garde S., Garcia A. E., Pohorille A., Pratt L. R., Proc. Nat. Acad. Sci. 93 (1996) 8951-8955.

[28] Morriss G. P., Daivis P. J., Evans D. J., J. Chem. Phys. 94 (1991) 7420-7433.

[29] Padilla P., Toxvaerd S., J. Chem. Phys. 97 (1992) 7687-7694.

[30] Mesli F., Mahboub R., Phys. Chem. News. 56 (2010) 117-127.

[31] Mesli F., Mahboub R., Mahboub M., Arab. J. Chem. 4 (2011) 211-222. 
[32] Mahboub R., Mahboub M., Mod. Appl. Sci. 6(5) (2012) 100-110.

[33] Mahboub R., Inter. Letters Chem. Phys. Astro. 5 (2012) 46-58.

[34] Mesli F., Mahboub R., Res. J. Pharm. Biol. Chem. Sci. 1(1) (2010) 83-92.

[35] van Gunsteren W. F., Berendsen H. J. C., Angew. Chem. Int. Ed. Engl. 29(9) (1990) 992-1023.

[36] Karplus M., Petsko G. A., Nature 347 (1990) 631-639.

[37] Sabbaghzadeh R., Monajjemi M., Mollaamin F., Oryan, S., J. Cell. Mol. Res. 2(2) (2010) 93-102. 